\title{
Ephemerides of the outer Jovian satellites
}

\author{
N. V. Emelyanov
}

\author{
Sternberg astronomical institute, 13 Universitetskij prospect, 119992 Moscow, Russia \\ e-mail: emelia@sai.msu.ru \\ Institut de mécanique céleste et de calcul des éphémérides - Observatoire de Paris, UMR 8028 du CNRS, \\ 77 avenue Denfert-Rochereau, 75014 Paris, France
}

Received 27 October 2004 / Accepted 20 January 2005

\begin{abstract}
The results of the determination of orbits for 54 outer satellites of Jupiter based on all available observations of these satellites are presented. The problem was solved using numerical integration method applied to the equations of satellite motion. After initial conditions of integration were refined, the residuals of observations from theory were obtained. Values of root-mean-square residuals for the eight satellites JVI-JXIII in right ascensions and declinations were found to be in the range $0.48^{\prime \prime}-0.67^{\prime \prime}$. The same parameters for the new outer Jovian satellites are in the range $0.10^{\prime \prime}-0.46^{\prime \prime}$. During the process of numerical integration of equations, the series based on the Chebychev polynomials for the satellite cordinates were defined. The series are used to calculate the apparent positions of satellites at the ephemerides. This ephemerides tool has broad capabilities including the possibility of obtaining residuals for a large series of user observations. The satellite motion model and numerical integration method adopted in this work were compared with the methods used at the Jet Propulsion Laboratory. This comparison of the apparent positions of satellites has shown that the differences between the two models are not greater than $0.012^{\prime \prime}$ in a 100-year interval. The residuals of satellite observations are similar to those obtained at JPL.
\end{abstract}

Key words. planets and satellites: general - planets and satellites: individual: Jupiter

\section{Introduction}

We are interested in the development of theories of natural satellite motion as well as determination of orbital parameters from observations. In this paper we restrict our consideration to the outer Jovian satellites.

Presently there are two independent tools for calculating satellite ephemerides. The numerical models of motion of the outer Jovian satellites built at the Jet Propulsion Laboratory (Jacobson 2000) on the basis of their observations and the corresponding ephemerides are available by Internet (Giorgini et al. 1996). Similar work has been carried out at the Institut de Mécanique Céleste et de Calcul des Ephémérides (IMCCE). However, the data of these two sources have noticeable differences.

In this paper we have carried out an independent numerical integration of the equations of motion of the outer Jovian satellites, as well as improvement of initial conditions based on observations. The corresponding ephemerides have been determined.

This work has been carried out using a calculating program that can perform both numerical integration and improvement of initial parameters. In the process of numerical integration, the Chebychev coefficients of satellite coordinates are calculated which are then used to compute the ephemerides.

We perform a numerical integration fitted to observations made from 1905 to April 15, 2004.
The results of our work are presented on the server of the planetary natural satellites ephemerides, http:// lnfm1. sai.msu.ru/neb/nss/index.htm. This web tool allows calculation of ephemerides of all natural satellites as well as to obtain differences between new observational data and the data obtained using models of satellite motion.

Our results may be compared to those obtained at JPL.

\section{The presentation of satellites}

The ephemerides of the 54 outer satellites of Jupiter including the new satellites discovered since 1999 were determined. Instead of standard designations for the named satellites where Roman numerals are used (JVI, JVII, etc.), we apply more simplified designations (J6, J7, etc.) These objects are moving at distances between 7 and 28 million kilometers from the planet. The distribution of the semimajor axes and eccentricities is shown in Fig. 1. The satellites Himalia (J6), Elara (J7), Lysithea (J10) and Leda (J13) are in direct orbits, their semimajor axes are between 11.16 and 11.74 million kilometers, while their inclinations with respect to the ecliptic are between 26 and 28 degrees. The satellite Themisto (J18) is in a direct orbit with a semimajor axis of 7.39 million kilometers and an inclination of 43.2 degrees. The satellite $\mathrm{S} / 2003 \mathrm{~J} 20$ is in a direct orbit with a semimajor axis of 17.14 million kilometers and an inclination of 51.4 degrees. Other satellites move in retrograde orbits with inclinations between 144 and 165 degrees. 


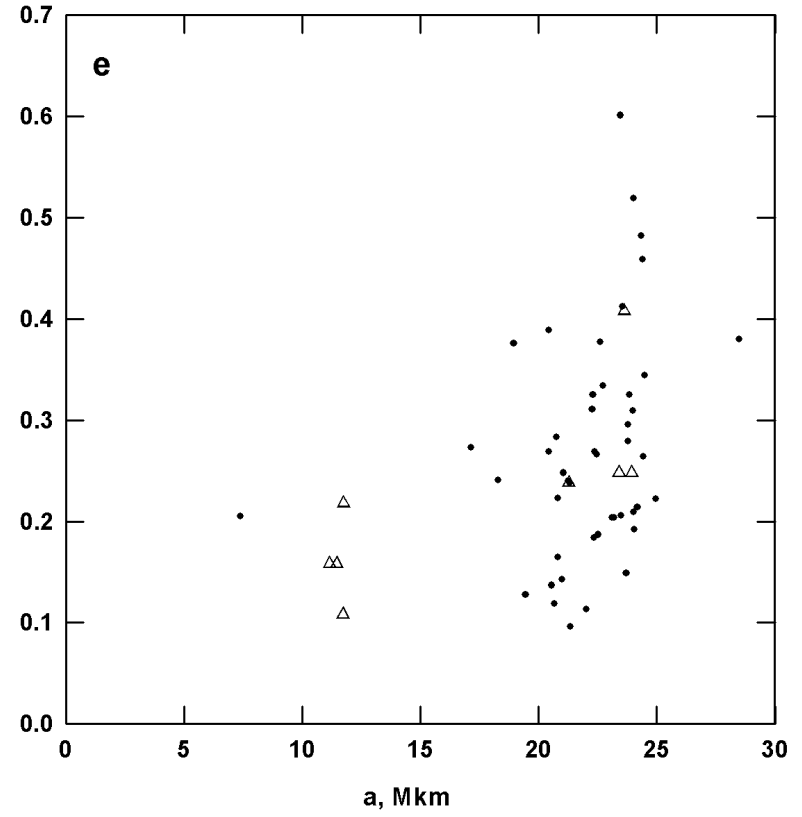

Fig. 1. The orbital parameter distribution ( $a-$ semimajor axis, $e-$ eccentricity) of the satellites J6-J13 (triangles) and other satellites (dots).

The masses of the faint satellites are uknown. Estimations of $G M$ based on assumed densities and on sizes based on magnitudes and assumed albedos are $0.63 \mathrm{~km}^{3} \mathrm{~s}^{-2}$ for Himalia (J6), $0.051 \mathrm{~km}^{3} \mathrm{~s}^{-2}$ for Elara $(\mathrm{J} 7)$, and rather less for other satellites (Simon 1997).

\section{Orbital motion model}

Representation of the orbits by analytical theory has proven to be quite difficult primarily because of the strong solar perturbation. High-precision ephemerides may be reliably produced only using numerical integration.

Our model for the orbits of the satellites is based on a numerical integration of their equations of motion in Cartesian Jovicentric coordinates referenced to the mean Earth equator and equinox of the J2000.0 system. These equations include perturbations from the Sun, Saturn, Uranus, Neptune and the effects of an oblate Jupiter ( $J 2$ and $J 4$ only). In our model the Galilean satellites were replaced by uniform circular equatorial rings. The mass of Jupiter is augmented by the mass of the Galilean satellites and corrected values of $J 2^{\prime}$ and $J 4^{\prime}$ are used for perturbations from the Galilean satellites. For $J 2^{\prime}$ and $J 4^{\prime}$ we have

$$
\begin{aligned}
& J 2^{\prime}=J 2+\frac{1}{2} \sum_{i=1}^{4} \frac{m_{i}}{m^{\prime}}\left(\frac{a_{i}}{R_{0}}\right)^{2}, \\
& J 4^{\prime}=J 4-\frac{3}{8} \sum_{i=1}^{4} \frac{m_{i}}{m^{\prime}}\left(\frac{a_{i}}{R_{0}}\right)^{4}, \\
& m^{\prime}=m_{0}+\sum_{i=1}^{4} m_{i},
\end{aligned}
$$

where $m_{0}$ is the mass of Jupiter; $R_{0}$ is its equatorial radius; $m_{i}(i=1,2,3,4)$ are the masses of the Galilean satellites; $a_{i}(i=1,2,3,4)$ are the radii of their orbits. We have adopted this simplified model of perturbations from the Galilean satellites as the differences between this model and the explicit perturbing satellites in resulting satellite coordinates are only of the order of $200 \mathrm{~km}$ (Jacobson 2000).

The mass of the Sun was augmented by the masses of Mercury, Venus, the Earth-Moon system and the Martian system to take into account part of the perturbing effects from the inner planets.

Because the outer satellites are small they may be assumed to be massless (Jacobson 2000).

The positions of the Sun, Saturn, Uranus and Neptune are from the JPL planetary ephemerides DE405.

Perturbations from the planet's non-sphericity are small so, according to our evaluations, we can neglect the precession of Jupiter's axis of rotation.

Values of all involved parameters were taken from Jacobson (2000). In such a case it is possible to compare our models of satellite motion. A revised gravity field for the Jovian system to use in the integration is available from Jacobson (2001). However, switching from the Jacobson (2000) system is not necessary as the changes are small.

\section{Observational basis and preliminary processing}

The ephemeride precision depends not only on the precision of observations but also on the period of the observations. Therefore all available observations should be used. A long data set is needed for orbit perturbation analysis in order to separate the secular perturbations from the long period perturbations.

It was not reasonable to compile a special complete catalogue for this work since a database of natural satellite observations exists: http://lnfm1.sai.msu.ru/neb/nss/ index.htm. It also includes all the data from the most advanced catalogue of observations used for the ephemerides of the eight outer Jovian satellites (Jacobson 2000). The observations used here are listed in Tables 1-3.

With regard to the new outer satellites of Jupiter we have used all the observations published in the International Astronomical Union Circulars (IAUC), the Minor Planet Electronic Circulars (MPEC), Minor Planet Center communucations to the Natural Satellites Data Center (IMCCE), and Minor Planet Circulars.

A detailed description of various series of observations as well as bibliographic references may be found in the abovementioned database.

As a preliminary processing for the observations referred to a mean equator and equinox of epoch system (other than the FK5/J2000), we first reduced the satellite positions to the FK4/B1950 system with the Newcomb precession constant and then reduced them to the FK5/J2000 system in accordance with the new IAU resolutions using the procedure described in Aoki et al. (1983). For the observations made prior to 1940 we applied an additional correction of 0.75 arcsec to all right ascensions as explained in Jacobson (2000). Our tests confirmed that this correction improves the fit to nearly all the early observations. 
Table 1. Observation residual statistics for satellites J6-J13.

\begin{tabular}{lccccccccccc}
\hline \hline & $\begin{array}{c}\text { Number of } \\
\text { positions } \\
\text { available }\end{array}$ & $\begin{array}{c}\text { Observa- } \\
\text { tion time } \\
\text { interval }\end{array}$ & $\begin{array}{c}\text { Number of } \\
\text { positions } \\
\text { used }\end{array}$ & $\begin{array}{c}\text { Mean } \\
r_{\alpha} \\
\left({ }^{\prime \prime}\right)\end{array}$ & $\begin{array}{c}\text { Mean } \\
r_{\delta} \\
\left({ }^{\prime \prime}\right)\end{array}$ & $\begin{array}{c}\text { rms } \\
\sigma_{\alpha} \\
\left({ }^{\prime \prime}\right)\end{array}$ & $\begin{array}{c}\text { rms } \\
\sigma_{\delta} \\
\left({ }^{\prime \prime}\right)\end{array}$ & $\begin{array}{c}\text { rmws } \\
\sigma_{\alpha}^{w} \\
\left({ }^{\prime \prime}\right)\end{array}$ & $\begin{array}{c}\text { rmws } \\
\sigma_{\delta}^{w} \\
\left({ }^{\prime \prime}\right)\end{array}$ & $\begin{array}{c}\text { rmws } \\
\sigma_{d}^{w} \\
\left({ }^{\prime \prime}\right)\end{array}$ \\
\hline J6 Himalia & 937 & $1905-2003$ & 852 & 0.042 & 0.071 & 0.843 & 0.779 & 0.627 & 0.580 & 0.854 \\
J7 Elara & 523 & $1905-2003$ & 449 & 0.132 & 0.020 & 0.875 & 0.928 & 0.651 & 0.668 & 0.933 \\
J8 Pasiphae & 838 & $1908-2003$ & 800 & 0.080 & 0.034 & 0.843 & 0.696 & 0.643 & 0.571 & 0.860 \\
J9 Sinope & 331 & $1914-2003$ & 316 & -0.020 & 0.124 & 0.842 & 0.706 & 0.764 & 0.637 & 0.994 \\
J10 Lysithea & 182 & $1938-2003$ & 173 & -0.047 & 0.142 & 0.751 & 0.608 & 0.632 & 0.532 & 0.826 \\
J11 Carme & 380 & $1938-2003$ & 355 & -0.008 & 0.233 & 0.724 & 0.711 & 0.648 & 0.669 & 0.931 \\
J12 Ananke & 227 & $1951-2003$ & 205 & 0.053 & 0.069 & 0.573 & 0.551 & 0.520 & 0.523 & 0.737 \\
J13 Leda & 77 & $1974-2003$ & 74 & 0.095 & 0.113 & 0.679 & 0.652 & 0.483 & 0.479 & 0.693 \\
\hline
\end{tabular}

Table 2. Observation residual statistics for satellites J17-J38 ( $T$ - time interval, $N$ - number of satellite positions available and used).

\begin{tabular}{lcccccc}
\hline \hline Satellite & $\begin{array}{c}\text { First observation } \\
\text { date }\end{array}$ & $\begin{array}{c}\text { Last observation } \\
\text { date }\end{array}$ & $\begin{array}{c}T \\
(\mathrm{~d})\end{array}$ & $\begin{array}{c}\sigma_{\alpha} \\
\left({ }^{\prime \prime}\right)\end{array}$ & $\begin{array}{c}\sigma_{\delta} \\
\left({ }^{\prime \prime}\right)\end{array}$ \\
\hline J17 Callirrhoe & 1999.10 & 2003.04 & 1302 & 47 & 0.26 & 0.26 \\
J18 Themisto & 1975.09 & 2003.04 & 10074 & 35 & 0.57 & 0.28 \\
J19 Megaclite & 2000.11 & 2003.02 & 825 & 38 & 0.25 & 0.22 \\
J20 Taygete & 2000.11 & 2003.03 & 852 & 50 & 0.25 & 0.31 \\
J21 Chaldene & 2000.11 & 2001.02 & 82 & 29 & 0.32 & 0.29 \\
J22 Harpalyke & 2000.11 & 2003.03 & 854 & 48 & 0.23 & 0.23 \\
J23 Kalyke & 2000.11 & 2003.04 & 885 & 64 & 0.37 & 0.44 \\
J24 Iocaste & 2000.11 & 2003.04 & 885 & 57 & 0.32 & 0.36 \\
J25 Erinome & 2000.11 & 2003.04 & 883 & 45 & 0.29 & 0.25 \\
J26 Isonoe & 2000.11 & 2003.04 & 885 & 57 & 0.27 & 0.35 \\
J27 Praxidike & 2000.11 & 2003.04 & 885 & 52 & 0.23 & 0.32 \\
J28 Autonoe & 2001.12 & 2003.04 & 503 & 26 & 0.36 & 0.34 \\
J29 Thyone & 2001.12 & 2003.03 & 471 & 24 & 0.23 & 0.20 \\
J30 Hermippe & 2001.12 & 2003.04 & 504 & 30 & 0.28 & 0.19 \\
J31 Aitne & 2001.12 & 2003.02 & 446 & 29 & 0.33 & 0.23 \\
J32 Eurydome & 2001.12 & 2003.03 & 473 & 23 & 0.20 & 0.21 \\
J33 Euanthe & 2001.12 & 2003.02 & 443 & 21 & 0.26 & 0.13 \\
J34 Euporie & 2001.12 & 2003.03 & 471 & 21 & 0.39 & 0.30 \\
J35 Orthosie & 2001.12 & 2003.03 & 471 & 23 & 0.21 & 0.26 \\
J36 Sponde & 2001.12 & 2003.02 & 445 & 16 & 0.27 & 0.27 \\
J37 Kale & 2001.12 & 2003.03 & 451 & 17 & 0.35 & 0.25 \\
J38 Pasithee & 2001.12 & 2003.02 & 444 & 17 & 0.26 & 0.22 \\
\hline
\end{tabular}

\section{From the numerical integration to production of ephemerides}

The numerical integration of the equations of motion and of the partial derivatives, the fit of orbits to observations and the representation of satellite coordinates with Chebyshev polynomials were made using original software.

To integrate the differential equations of satellite motion we used the method of Belikov (1993). It is an efficient single-step integrator which uses a uniform approximation of the solution and the truncated Chebyshev series of arbitrary order. The integration was carried out with a variable step size.

During the integration process the series in terms of the Chebyshev coefficients were built representing each satellite's rectangular coordinates. For all satellites, the approximation interval was chosen to be 20 days; while the polynomial degree was 13.
We used a least-squares procedure to fit the orbits to observations by adjusting the epoch state vectors of the integrated orbit.

The ephemerides based on the orbits described in this paper are available electronically as part of the Natural Satellites Service (NSS).

\section{The fit of the numerical model}

All outer Jovian satellites were divided into several groups. For each group calculations were made separately. Using preassigned initial conditions, numerical integration of both the equations of motion for each group and of the equations for the corresponding partial derivative were performed. The intervals of integration were taken differently for each group. For the satellites J6-J13 the integration started from October 1.0, 1905. For the new satellites, the initial date of integration 
Table 3. Observation residual statistics for the new unnumbered satellites ( $T$ - time interval, $N$ - number of satellite position available and used).

\begin{tabular}{|c|c|c|c|c|c|c|}
\hline Satellite & $\begin{array}{c}\text { First observation } \\
\text { date }\end{array}$ & $\begin{array}{c}\text { Last observation } \\
\text { date }\end{array}$ & $\begin{array}{c}T \\
\text { (d) }\end{array}$ & $N$ & $\begin{array}{l}\sigma_{\alpha} \\
\left({ }^{\prime \prime}\right)\end{array}$ & $\begin{array}{l}\sigma_{\delta} \\
\left({ }^{\prime \prime}\right)\end{array}$ \\
\hline $\mathrm{S} / 2002 \mathrm{~J} 1$ & 2002.10 & 2003.12 & 420 & 29 & 0.28 & 0.30 \\
\hline $\mathrm{S} / 2003 \mathrm{~J} 1$ & 2003.02 & 2004.04 & 435 & 24 & 0.37 & 0.43 \\
\hline $\mathrm{S} / 2003 \mathrm{~J} 2$ & 2003.02 & 2003.03 & 27 & 8 & 0.16 & 0.11 \\
\hline $\mathrm{S} / 2003 \mathrm{~J} 3$ & 2003.02 & 2003.03 & 26 & 15 & 0.23 & 0.20 \\
\hline $\mathrm{S} / 2003 \mathrm{~J} 4$ & 2003.02 & 2003.03 & 27 & 11 & 0.14 & 0.19 \\
\hline $\mathrm{S} / 2003 \mathrm{~J} 5$ & 2003.02 & 2003.04 & 78 & 22 & 0.32 & 0.30 \\
\hline $\mathrm{S} / 2003 \mathrm{~J} 6$ & 2003.02 & 2003.12 & 322 & 33 & 0.34 & 0.30 \\
\hline $\mathrm{S} / 2003 \mathrm{~J} 7$ & 2002.01 & 2003.06 & 506 & 33 & 0.25 & 0.36 \\
\hline $\mathrm{S} / 2003 \mathrm{~J} 8$ & 2002.01 & 2003.04 & 471 & 23 & 0.26 & 0.28 \\
\hline $\mathrm{S} / 2003 \mathrm{~J} 9$ & 2003.02 & 2003.03 & 49 & 17 & 0.26 & 0.36 \\
\hline $\mathrm{S} / 2003 \mathrm{~J} 10$ & 2003.02 & 2003.03 & 29 & 11 & 0.34 & 0.51 \\
\hline $\mathrm{S} / 2003 \mathrm{~J} 11$ & 2003.02 & 2003.12 & 322 & 17 & 0.42 & 0.44 \\
\hline $\mathrm{S} / 2003 \mathrm{~J} 12$ & 2003.02 & 2003.03 & 27 & 11 & 0.22 & 0.19 \\
\hline $\mathrm{S} / 2003 \mathrm{~J} 13$ & 2002.12 & 2004.04 & 471 & 22 & 0.21 & 0.40 \\
\hline $\mathrm{S} / 2003 \mathrm{~J} 14$ & 2003.02 & 2003.04 & 53 & 11 & 0.17 & 0.20 \\
\hline $\mathrm{S} / 2003 \mathrm{~J} 15$ & 2003.02 & 2003.04 & 56 & 12 & 0.22 & 0.25 \\
\hline $\mathrm{S} / 2003 \mathrm{~J} 16$ & 2003.02 & 2003.04 & 56 & 12 & 0.16 & 0.15 \\
\hline $\mathrm{S} / 2003 \mathrm{~J} 17$ & 2003.02 & 2003.04 & 54 & 12 & 0.15 & 0.15 \\
\hline $\mathrm{S} / 2003 \mathrm{~J} 18$ & 2003.02 & 2003.04 & 78 & 20 & 0.21 & 0.19 \\
\hline $\mathrm{S} / 2003 \mathrm{~J} 19$ & 2003.02 & 2003.04 & 56 & 10 & 0.12 & 0.25 \\
\hline $\mathrm{S} / 2003 \mathrm{~J} 20$ & 2003.02 & 2004.01 & 349 & 37 & 0.21 & 0.30 \\
\hline $\mathrm{S} / 2003 \mathrm{~J} 21$ & 2002.01 & 2003.04 & 472 & 28 & 0.26 & 0.21 \\
\hline $\mathrm{S} / 2003 \mathrm{~J} 22$ & 2002.01 & 2003.04 & 471 & 25 & 0.31 & 0.32 \\
\hline $\mathrm{S} / 2003 \mathrm{~J} 23$ & 2003.02 & 2003.03 & 29 & 16 & 0.39 & 0.30 \\
\hline
\end{tabular}

was taken from the first observation date. For each satellite the dates of the first observation used are given in Tables 2 and 3 . Integration of the equations of motion was carried out up to the final date of the ephemerides. For J6-J13 this date is October 7.0, 2025. For the other satellite groups different final dates in 2027 have been taken.

For each observation date, residuals of observations from theory and conditional equation coefficients were computed. After integration, conditional equations were solved by a least squares fit and corrections to the initial conditions were obtained. After that the process was repeated.

Improvement of the initial conditions was stopped when the corrections were 50 times less than their errors defined by a least squares fit. After the process was completed, the last version of Chebyshev polynomials for the rectangular planetocentric coordinates of each satellite was stored for subsequent ephemerides calculation.

For the satellites J6-J13, for some sets of early observational results determination of right ascension and declination biases was included. If a bias turned out to be less than 1 arcsec, it was put to zero. This minimizes the residuals and excludes possible systematic errors.

For the satellites J6-J13, weights were assigned to each observation set according to the observer and observation epoch. A number of observations of these satellites were rejected at the stages of both preliminary analysis and of the orbital fit, when residuals in right ascension and declination exceeded 4 arcsec.
The number of observed positions used for these satellites is given in Table 1.

For the satellites J17-J38 and unnumbered satellites no observation was rejected and all positions were assumed to be of the same precision. The number of observed positions used for each satellite is given in Tables 2 and 3.

The epoch state vectors from the fit of the orbits to the observations for all the satellites are presented at http://lnfm1.sai.msu.ru/neb/nss/index.htm.

\section{Analysis}

The observation residual statistics for the eight satellites J6-J13 are shown in Table 1. Since weights were assigned to these satellites, the root mean weighted squares (rmws) and the root mean squares (unweighted) are given in Table 1.

Since for all the satellites the mean values of residuals in right ascension and declination turned out to be significantly less than the corresponding rms, we may conclude that the systematic errors of observations are either negligible or were removed after biases were taken into consideration.

For other satellites the evaluations are given in Tables 2 and 3. No systematic errors were found for the observations of these satellites.

To check the ephemerides produced with our Natural Satellites Service (NSS) based on these orbits, a comparison was made with the JPL ephemerides. The JPL ephemerides 




Fig. 2. JPL ephemerides deviations from NSS ephemerides for satellite J6 Himalia (circles - in right ascension, crosses - in declination).

were generated via the Internet using the Horizons system (Giorgini et al. 1996).

We compared the two independent models of satellite motion. For this we used the Horizons system to generate right ascension and declination values for the satellites J6-J13 in interval 1905-2005 with a step of 20 days. We then improved the initial conditions of integration using our calculating program. Residuals obtained in this way may be caused only by the differences in the two models of satellite motion since there are no observational errors in this case. For these eight satellites, $\mathrm{J} 6-\mathrm{J} 13$, residuals in right ascension and declination did not exceed $0.012^{\prime \prime}$. Rms residuals proved to be $0.007^{\prime \prime}$ in both right ascension and declination. These results show that the disagreement between the JPL and NSS models is significantly less than the errors in the available observations.

For the satellites J6-J13 the ephemerides were generated in the time interval, from 1950 to 2020 . For other satellites we made a comparison in the time interval of available observations.

In Fig. 2 an example of the disagreement between the JPL ephemerides and ours is seen for Himalia (J6). Such disagreements are considerably less than the observational errors and may be accounted for by the different observation sets used at JPL and in our work.

Figures 3-6 display examples of residuals for some of the new outer satellites of Jupiter. In these figures we show the residuals of the JPL ephemerides from our results. It is seen that the disagreement between the two ephemeride sources is significantly less than the observational errors.

The analysis of observational errors for each single observatory is of particular interest. We have combined residuals for the eight satellites J6-J13 for each observatory. The mean values of residuals and corresponding rms in right ascension and declination have been calculated. The results are given in Table 4. For the early observations some biases have been included. In Table 4 the number of separate time intervals is given for which biases were introduced. From the data in the Table 4 it is seen that the precisions of observations made at different observatories significantly differ. Note the high precision and

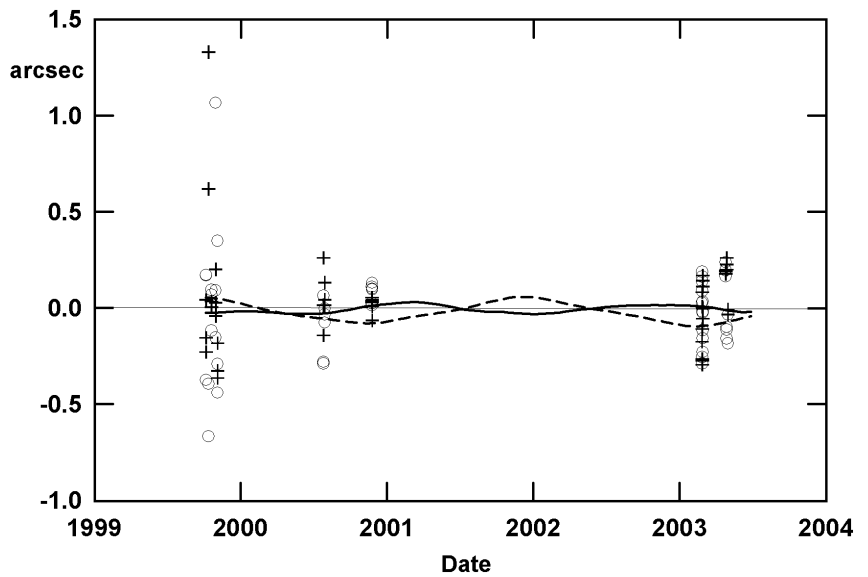

Fig. 3. Residuals (O-C) for satellite J17 Callirrhoe and JPL ephemerides deviations (circles - $\mathrm{O}-\mathrm{C}$ in right ascension, crosses - $\mathrm{O}-\mathrm{C}$ in declination, line - JPL-NSS in right ascension, dashed line - JPL-NSS in declination).



Fig. 4. Residuals (O-C) for satellite J18 Themisto and JPL ephemerides deviations (circles - $\mathrm{O}-\mathrm{C}$ in right ascension, crosses $-\mathrm{O}-\mathrm{C}$ in declination, line - JPL-NSS in right ascension, dashed line - JPL-NSS in declination).

absence of systematic errors in observations made at observatory 689 - U.S.N.O. Flagstaff in 1998-2001.

Among the observations that were not used in the paper (Jacobson 2000) and which make a large contribution to the observational basis are data from the observatories 94 - CrimeaSimeis (78 positions) and 115 - Zelenchukskaya (84 positions). These observations have good precision.

Compared with the results of Jacobson (2000) the ephemerides of the satellites J6-J13 are improved in our work through the inclusion of more observations.

Observations of the new outer Jovian satellites have been made using powerful telescopes at several observatories. All these observations have roughly the same precision.

\section{Conclusions}

We have elaborated ephemerides for all outer satelites of Jupiter including 46 new satellites found before September 2004. These ephemerides are based on all available 
Table 4. Residual statistics summary for satellites J6-J13 by observatories (N pos. - number of satellite positions used, N bias - number of oppositions with biases applied).

\begin{tabular}{|c|c|c|c|c|c|c|c|c|}
\hline $\begin{array}{l}\text { Obs. } \\
\text { code } \\
\text { MPC }\end{array}$ & $\begin{array}{l}\text { Observatory } \\
\text { name }\end{array}$ & $\begin{array}{l}\text { Periods of } \\
\text { observation }\end{array}$ & $\begin{array}{c}\mathrm{N} \\
\text { pos. }\end{array}$ & $\begin{array}{c}\text { Mean } \\
\overline{r_{\alpha}} \\
\left({ }^{\prime \prime}\right)\end{array}$ & $\begin{array}{c}\text { Mean } \\
\overline{r_{\delta}} \\
\left({ }^{\prime \prime}\right)\end{array}$ & $\begin{array}{c}\mathrm{rms} \\
\sigma_{\alpha} \\
\left({ }^{\prime \prime}\right)\end{array}$ & $\begin{array}{c}\mathrm{rms} \\
\sigma_{\delta} \\
\left({ }^{\prime \prime}\right)\end{array}$ & $\begin{array}{c}\mathrm{N} \\
\text { bias }\end{array}$ \\
\hline 0 & Greenwich & $1905-1910$ & 204 & -0.01 & 0.00 & 1.07 & 0.84 & 6 \\
\hline 24 & Heidelberg-Konigstuhl & 1906-1909, 1930 & 14 & 0.03 & -0.02 & 1.02 & 1.59 & 3 \\
\hline 29 & Hamburg-Bergedorf & 1932,1933 & 15 & -0.09 & -0.18 & 1.80 & 1.05 & 1 \\
\hline 87 & Helwan & 1911-1914, 1916-1922 & 80 & 0.03 & -0.02 & 1.06 & 0.96 & 3 \\
\hline 94 & Crimea-Simeis & 2001-2002 & 78 & 0.00 & 0.19 & 0.40 & 0.48 & - \\
\hline 106 & Crni Vrh & 2000 & 3 & -0.46 & 0.50 & 0.43 & 0.51 & - \\
\hline 115 & Zelenchukskaya & 1999-2000 & 84 & 0.07 & 0.21 & 0.33 & 0.35 & - \\
\hline 185 & Jurassien-Vicques & 2002 & 4 & -0.24 & 0.32 & 0.34 & 0.32 & - \\
\hline 210 & Alma-Ata & 1952 & 3 & 0.16 & -0.96 & 1.19 & 1.62 & - \\
\hline 261 & Palomar Mountain-DSS & 1974 & 6 & 0.01 & 0.05 & 1.35 & 0.81 & 1 \\
\hline 291 & LPL/Spacewatch II & 2003 & 39 & 0.04 & 0.12 & 0.25 & 0.19 & - \\
\hline 333 & Desert Eagle Observatory & 2003 & 8 & 0.16 & -0.12 & 0.25 & 0.19 & - \\
\hline 365 & Uto Observatory & 1993 & 6 & -0.00 & -0.01 & 1.35 & 1.08 & 1 \\
\hline 381 & Tokyo-Kiso & 1986-1992 & 207 & 0.09 & 0.01 & 0.77 & 0.67 & - \\
\hline 413 & Siding Spring Observatory & 1993 & 5 & -0.46 & 0.02 & 1.89 & 0.58 & - \\
\hline 568 & Mauna Kea & 2000,2003 & 81 & -0.28 & 0.11 & 0.61 & 0.28 & - \\
\hline 608 & Haleakala-AMOS & 2001-2003 & 96 & 0.14 & 0.21 & 0.68 & 0.68 & - \\
\hline 636 & Essen & 2002 & 2 & -0.64 & 0.79 & 0.82 & 0.99 & - \\
\hline 644 & Palomar Mountain/NEAT & $2001-2003$ & 123 & 0.19 & 0.39 & 0.40 & 0.55 & - \\
\hline 660 & Leuschner Obs., Berkeley & 1951 & 6 & -0.23 & -0.02 & 0.33 & 0.24 & - \\
\hline 662 & Lick Obs., Mount Hamilton & 1905-1914, 1934-1969 & 245 & 0.05 & -0.05 & 1.29 & 1.35 & 6 \\
\hline 672 & Mount Wilson & 1916-1951 & 174 & -0.24 & 0.11 & 1.10 & 0.97 & 4 \\
\hline 673 & Table Mountain & $1968,1997-1999$ & 18 & 0.01 & -0.23 & 0.36 & 0.39 & - \\
\hline 675 & Palomar Mountain & 1976 & 2 & -0.70 & -0.85 & 0.68 & 1.13 & - \\
\hline 689 & U.S.N.O., Flagstaff & 1998-2001 & 340 & 0.01 & -0.09 & 0.21 & 0.26 & - \\
\hline 690 & Lowell Observatory, Flagstaff & $1934,1958-1965,1980-1981$ & 74 & 0.21 & 0.14 & 1.02 & 0.82 & 3 \\
\hline 691 & Steward Observatory, Kitt Peak & $1975,1992-1993,2000-2003$ & 49 & 0.10 & -0.05 & 0.44 & 0.45 & 1 \\
\hline 693 & Catalina Station, Tucson & 1975 & 4 & 0.76 & 0.29 & 1.18 & 0.39 & - \\
\hline 695 & Kitt Peak & 1974 & 4 & 0.01 & 0.07 & 0.59 & 0.22 & 1 \\
\hline 696 & Whipple Obs., Mt. Hopkins & 2001 & 13 & -0.01 & 0.09 & 0.25 & 0.19 & - \\
\hline 699 & Lowell Observatory-LONEOS & 1998 & 5 & 0.00 & -0.00 & 2.21 & 0.89 & 1 \\
\hline 703 & Catalina Sky Survey & 2003 & 20 & 0.76 & 0.17 & 0.87 & 0.71 & - \\
\hline 704 & Lincoln Lab., NewMexico & $2000-2003$ & 481 & 0.03 & 0.25 & 0.73 & 0.66 & - \\
\hline 711 & McDonald Obs., Fort Davis & 1944-1954, 1995 & 540 & 0.14 & 0.05 & 0.63 & 0.64 & 2 \\
\hline 732 & Oaxaca & 2000 & 2 & -0.81 & -0.14 & 0.76 & 0.15 & - \\
\hline 754 & Yerkes Obs., Williams Bay & 1906-1923, 1930-1934, 1954 & 47 & 0.50 & 0.10 & 1.55 & 1.05 & - \\
\hline 760 & Goethe Link Obs., Brooklyn & 1970 & 2 & 0.04 & 0.01 & 0.39 & 0.29 & 1 \\
\hline 786 & U.S.N.O., Washington & 1905-1919, 1927, 1941 & 46 & -0.01 & 0.00 & 1.12 & 0.89 & 5 \\
\hline 807 & Cerro Tololo, La Serena & 1998 & 4 & 0.22 & 0.09 & 0.51 & 0.12 & - \\
\hline 809 & ESO, La Silla & 1992-1993, 2000 & 41 & 0.17 & 0.11 & 0.65 & 0.66 & - \\
\hline 822 & Cordoba & 1938-1939, 1952, 1965 & 17 & -0.03 & -0.07 & 0.93 & 0.81 & - \\
\hline 835 & Drum Hill Station & 1999 & 9 & 0.28 & -0.17 & 0.64 & 0.66 & - \\
\hline 938 & Linhaceira & 2003 & 1 & -0.50 & 0.54 & 0.48 & 0.54 & - \\
\hline 950 & La Palma & 2002 & 2 & -0.21 & 0.03 & 0.19 & 0.03 & - \\
\hline 999 & Bordeaux-Floirac & 1967 & 14 & -0.40 & 0.87 & 0.72 & 0.96 & - \\
\hline $\mathrm{H} 29$ & Ivywood Obs., Edmond & 2003 & 6 & -0.39 & 0.08 & 0.79 & 0.64 & - \\
\hline
\end{tabular}

ground-based observations. In the theory of satellite motion all necessary perturbations were taken into account, which provides precision of topocentric positions within $0.01^{\prime \prime}$. The real precision of satellite coordinates is restricted to the observational precision.

The ephemerides of the satellites are available at: http://lnfm1. sai.msu.ru/neb/nss/index .htm.
Comparison of the adopted model of satellite motion with that of JPL reveals that the precision of the adopted model is at least 50 times better than the precision of present-day observations.

Reliability and precision of the ephemerides depend not only on the observational precision but also on the time interval during which the satellite was observed. At present, 




Fig. 5. Residuals (O-C) for satellite J23 Kalyke and JPL ephemerides deviations (circles $-\mathrm{O}-\mathrm{C}$ in right ascension, crosses $-\mathrm{O}-\mathrm{C}$ in declination, line - JPL-NSS in right ascension, dashed line - JPL-NSS in declination).

the intervals of observation of the new outer satellites of giant planets are not large. Some of the intervals of observations are less than one orbital period. Any new observations will improve the precision of the ephemerides.

For the satellites J6-J13 the ephemerides may be calculated with our ephemeride server for the interval 1905-2025. The real precision of the ephemerides is not worse than $0.2^{\prime \prime}$.

Presently, the dates for which ephemerides of the new Jovian satellites may be calculated may be taken beginning from the first observation date to 2027. For the period 2004-2006 the real precision of the ephemerides corresponds to the observational precision and is about $0.5^{\prime \prime}$.

The following possibilities are offered:

- Calculation of astrometric, geocentric or topocentric coordinates of a satellite for any given date. Calculation of differences in coordinates of a planet and satellite or of two satellites is also possible.

- Calculation of a satellite's rectangular planetocentric coordinates and velocities.

- Calculation of osculating elements of a satellite's Keplerian orbit for any given date.

- Calculation of ephemerides for a sequence of arbitrary dates given in tabular form.

- Calculation of residuals of observational results from the ephemerides (“O-C”). In this case observational results are

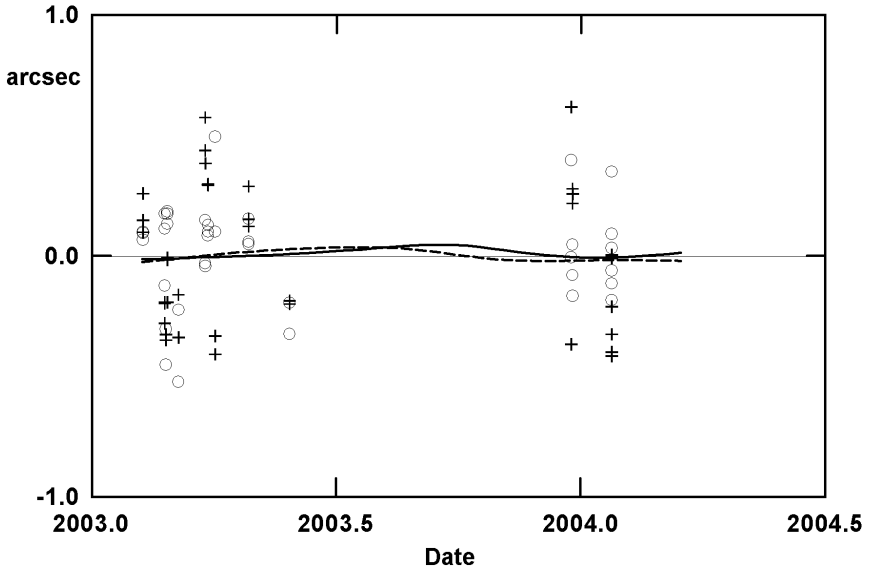

Fig. 6. Residuals (O-C) for satellite S/2003 J20 and JPL ephemerides deviations (circles $-\mathrm{O}-\mathrm{C}$ in right ascension, crosses $-\mathrm{O}-\mathrm{C}$ in declination, line - JPL-NSS in right ascension, dashed line - JPL-NSS in declination).

entered in tabular form and when the residuals are given the statistical characteristics of the observational errors are also displayed.

The Natural Satellites Service developed at the Celestial Mechanics Department of the Sternberg Astronomical Institute of Moscow State University will update the models of satellite motion and improve ephemerides precision as new observations become available.

Acknowledgements. This work was supported by the Russian Foundation for Basic Research, project No. 03-02-17441.

\section{References}

Aoki, S., Soma, M., Kinoshita, H., \& Inoue, K. 1983, A\&A, 129, 263

Belikov, M. V. 1993, Manuscripta geodaetica, 18, 182

Giorgini, J. D., Yeomans, D. K., Chamberlin, A. B., et al. 1996, BAAS, 28, No. 25.04

Simon, J.-L., Chapront-Touzé, M., Morando, B., \& Thuillot, W. 1997, Introduction aux éphémérides astronomiques, Supplément explicatif à la connaissance des temps (Les Ulis: EDP Sciences)

Jacobson, R. A. 2000, AJ, 120, 2679

Jacobson, R. A. 2001, BAAS, 33, 1039 